\title{
Three Dimensional Measurement of Ideal Trajectory of Pedicle Screws of Subaxial Cervical Spine Using the Algorithm Could Be Applied for Robotic Screw Insertion
}

\author{
Jisoon Huh, ${ }^{1}$ Jae Hwan Hyun, ${ }^{1}$ Hyeong Geon Park, ${ }^{1}$ Ho-Young Kwak ${ }^{2}$ \\ Department of Neurosurgery, Jeju National University School of Medicine, Jeju, Korea \\ Department of Computer Engineering, ${ }^{2}$ Jeju National University College of Engineering, Jeju, Korea
}

Objective : To define optimal method that calculate the safe direction of cervical pedicle screw placement using computed tomography (CT) image based three dimensional (3D) cortical shell model of human cervical spine.

Methods : Cortical shell model of cervical spine from C3 to C6 was made after segmentation of in vivo CT image data of 44 volunteers. Three dimensional Cartesian coordinate of all points constituting surface of whole vertebra, bilateral pedicle and posterior wall were acquired. The ideal trajectory of pedicle screw insertion was defined as viewing direction at which the inner area of pedicle become largest when we see through the biconcave tubular pedicle. The ideal trajectory of 352 pedicles (eight pedicles for each of 44 subjects) were calculated using custom made program and were changed from global coordinate to local coordinate according to the three dimensional position of posterior wall of each vertebral body. The transverse and sagittal angle of trajectory were defined as the angle between ideal trajectory line and perpendicular line of posterior wall in the horizontal and sagittal plane. The averages and standard deviations of all measurements were calculated.

Results : The average transverse angles were $50.60^{\circ} \pm 6.22^{\circ}$ at $\mathrm{C} 3,51.42^{\circ} \pm 7.44^{\circ}$ at $\mathrm{C} 4,47.79^{\circ} \pm 7.61^{\circ}$ at $\mathrm{C} 5$, and $41.24^{\circ} \pm 7.76^{\circ}$ at $\mathrm{C} 6$. The transverse angle becomes more steep from $\mathrm{C} 3$ to $\mathrm{C} 6$. The mean sagittal angles were $9.72^{\circ} \pm 6.73^{\circ}$ downward at $\mathrm{C} 3,5.09^{\circ} \pm 6.39^{\circ}$ downward at $\mathrm{C} 4,0.08^{\circ} \pm 6.06^{\circ}$ downward at $\mathrm{C} 5$, and $1.67^{\circ} \pm 6.06^{\circ}$ upward at $\mathrm{C} 6$. The sagittal angle changes from caudad to cephalad from $\mathrm{C} 3$ to $\mathrm{C} 6$.

Conclusion : The absolute values of transverse and sagittal angle in our study were not same but the trend of changes were similar to previous studies. Because we know 3D address of all points constituting cortical shell of cervical vertebrae. we can easily reconstruct 3D model and manage it freely using computer program. More creative measurement of morphological characteristics could be carried out than direct inspection of raw bone. Furthermore this concept of measurement could be used for the computing program of automated robotic screw insertion.

Key Words : Cervical vertebrae · Computer-assisted image analysis · Pedicle screws · Three dimensional images.

- Received : September 3, 2018 •Revised : October 10, 2018 •Accepted : December 24, 2018

- Address for reprints : Ho-Young Kwak

Department of Computer Engineering, Jeju National University College of Engineering, 102 Jejudaehak-ro, Jeju 63243, Korea

Tel : +82-64-754-3653, Fax : +82-64-755-3620, E-mail : hykwak@gmail.com, ORCID : https://orcid.org/0000-0001-9438-7573

This is an Open Access article distributed under the terms of the Creative Commons Attribution Non-Commercial License (http://creativecommons.org/licenses/by-nc/4.0) which permits unrestricted non-commercial use, distribution, and reproduction in any medium, provided the original work is properly cited. 


\section{INTRODUCTION}

Cervical pedicle is a tiny and strongest tubular structure that can provide three column fixation like thoracic and lumbar vertebrae. It is well known that cervical pedicle screw fixation supplies biomechanically stronger fixating power and higher pullout strength among posterior cervical fixation ${ }^{4,6)}$. Cervical pedicle has complex anatomy neighboring vulnerable vascular and neural structures and catastrophic dangers could be encountered when screws penetrate theses small structure. The spine surgeons are unfamiliar to insert pedicle screws with blunt techniques because of its invisibility and difficulty in identifying its marginal contour during posterior approach. There are numerous studies to reveal the anatomical characteristics of cervical pedicle to aid safe pedicle fixation. The previous studies used various sources to measure from direct visualization of raw bone ${ }^{10)}, \mathrm{X}$-rays ${ }^{13)}$, computed tomography (CT) images ${ }^{14)}$ and three dimensional reconstruction images ${ }^{12)}$
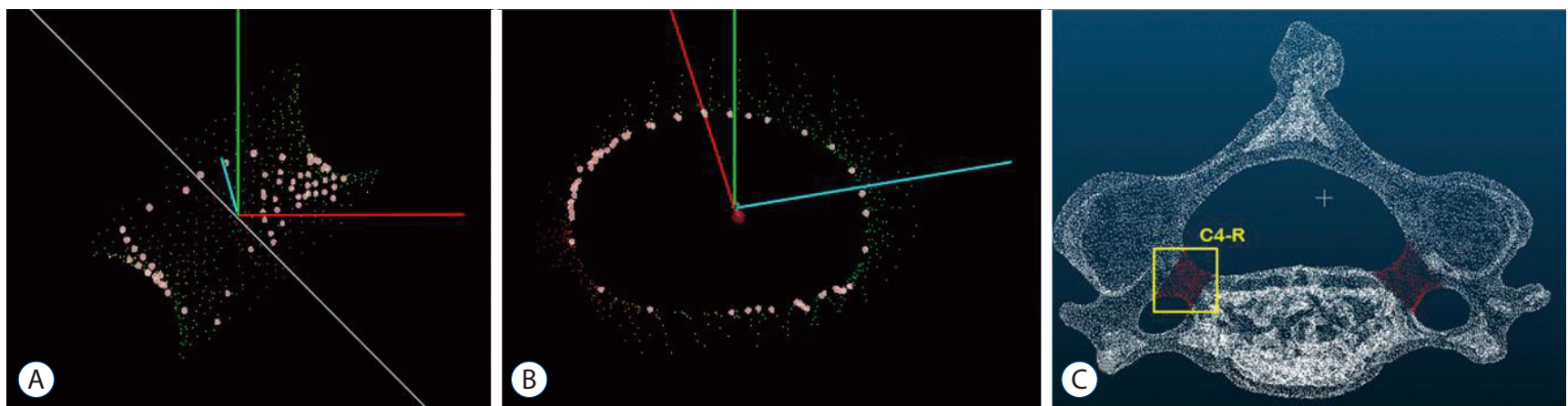

Fig. 1. Ideal trajectory of pedicle was defined as viewing direction (A) at which the inner area of pedicle become largest (B) when we see through the biconcave tubular pedicle (C).

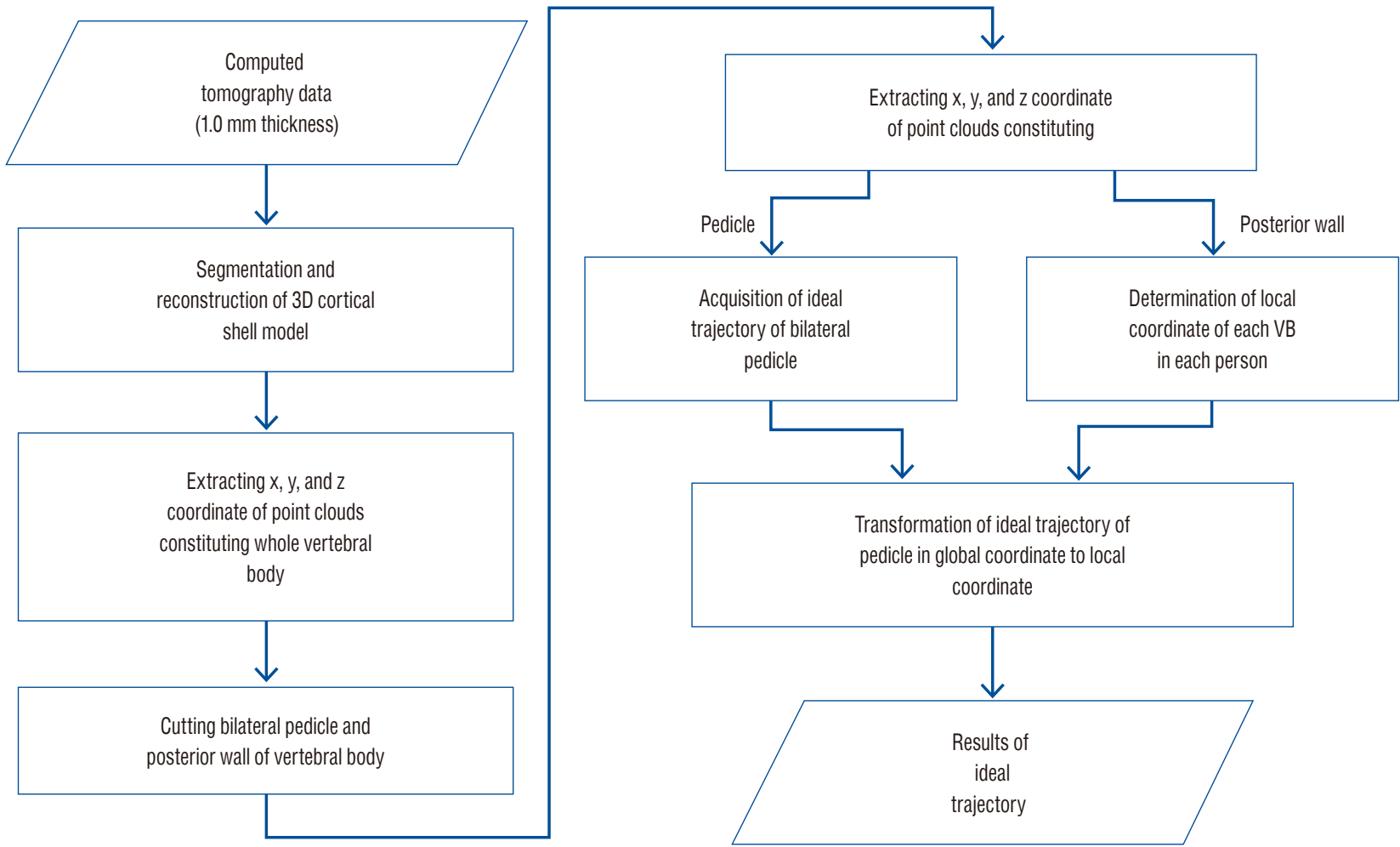

Fig. 2. Algorithm for calculating ideal trajectory of cervical pedicle screw insertion from computed tomography image data. VB: vertebral body. 
and analyzed linear and angular dimension of cervical pedi$\mathrm{cle}^{7}$, relationship with surrounding neural and vascular structures $^{16)}$, and optimal entry point of pedicle screws ${ }^{5,9}$. Although differing to various clinical conditions that could lead to any deformity, studies for complications of cervical pedicle screw placement revealed high penetration rate in freehand technique. Any guidance techniques or devices including navigation or computer assisted placement were recommended in spite of the price limit ${ }^{3}$.

The aim of this study is to define optimal method to calculate the safe direction of pedicle screw placement using computed tomography image based three dimensional cortical shell model of human cervical spine.

\section{MATERIALS AND METHODS}

Total 44 volunteers (mean age, 35.2 years [range, 20-62]; mean weight, $64.0 \mathrm{~kg}$ [range, 46.3-89.9]; and mean height, $165.7 \mathrm{~cm}$ [range, 147.2-186.0]) were enrolled in this study (Institutional Review Board of Jeju National University Hospital approved) and underwent axial cervical CT scanning at 1.0$\mathrm{mm}$ slice thickness. The CT image data of each subject were reviewed by the authors for preexisting cervical spine pathology to exclude from this study. Exclusion criteria for this study were history of spinal surgery, age under 20 years and over 65 years, present radiating pain, extreme obesity, any contraindications to magnetic resonance and CT imaging, severe osteoporosis, disc collapse at multiple levels, severe spinal stenosis, congenital spinal pathology, and any destructive process involving the spine. Image data in a Digital Imaging and Communication in Medicine format from the level $\mathrm{C} 1$ to $\mathrm{C} 7$ were acquired from the CT scanner (Somatom definition, Siemens, Malvern, PA, USA).

Well trained medical students reconstructed cortical shell model from C3 to C6 using a three-dimensional reconstruction software package (Mimics, Materialise Inc., Leuven, Belgium). The $\mathrm{x}, \mathrm{y}$, and $\mathrm{z}$ coordinate of points that constitute the cortex of whole vertebral body were acquired. The bilateral pedicle and posterior wall of each vertebral body were cut using open source software (CloudCompare [Free Software Foundation, Boston, MA, USA], 3D point cloud and mesh processing software [Free Software Foundation]). The point cloud data constituting each pedicle and posterior wall were also obtained. The ideal trajectory of pedicle screw placement was defined as viewing direction at which the inner area of pedicle become largest when we see through the biconcave tubular pedicle (Fig. 1).

Total 352 (eight pedicles from C3 to C6 bilaterally for each of 44 subjects) ideal trajectory were calculated using custom made program (made by H.Y.K.).

The position of vertebral body is different in each person.

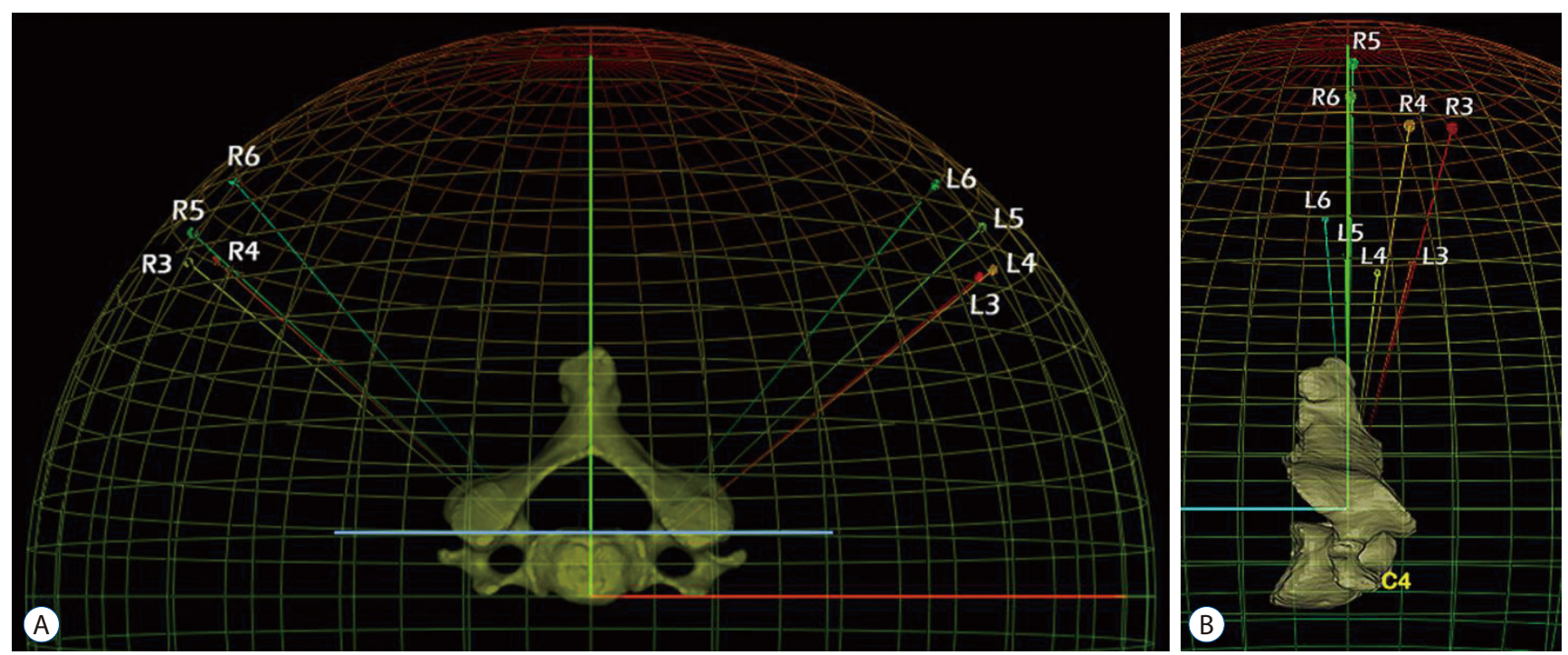

Fig. 3. The transverse (A) and sagittal (B) angle of trajectory were defined as the angle between calculated trajectory and perpendicular line of posterior wall of vertebral body in the horizontal and sagittal plane. $R$ : right, L : left. 
The standardization of pedicle trajectory was needed to compare among each person by the level of concern. The standardized new $\mathrm{x}, \mathrm{y}, \mathrm{z}$ Cartesian eigen vectors that represent the local coordinate of each vertebral body were calculated using principal component analysis of point cloud data of posterior wall and each whole vertebral body, The ideal trajectory of pedicle screw placement in global CT coordinate were transformed using direction cosine matrix to local coordinate of each vertebral body (Fig. 2).

The transverse and sagittal angle of trajectory were defined as the angle between ideal trajectory line and perpendicular line of posterior wall of vertebral body in the horizontal and sagittal plane (Fig. 3). The averages and standard deviations of all measurements were calculated (Tables 1,2).

\section{RESULTS}

The average transverse angles were $50.60^{\circ} \pm 6.22^{\circ}$ at C3, $51.42^{\circ} \pm 7.44^{\circ}$ at $\mathrm{C} 4,47.79^{\circ} \pm 7.61^{\circ}$ at $\mathrm{C} 5$, and $41.24^{\circ} \pm 7.76^{\circ}$ at C6. The transverse angle becomes more steep from C3 to C6.

The mean sagittal angles were $9.72^{\circ} \pm 6.73^{\circ}$ downward at $\mathrm{C} 3$, $5.09^{\circ} \pm 6.39^{\circ}$ downward at C4, $0.08^{\circ} \pm 6.06^{\circ}$ downward at C5, and $1.67^{\circ} \pm 6.06^{\circ}$ upward at $\mathrm{C} 6$. The sagittal angle changes from caudad to cephalad from C3 to C6.

\section{DISCUSSION}

The reference planes for angular measurement in human body part especially vertebra are so varied and vague. Most studies for transverse angle of cervical pedicle used anatomical sagittal plane as the reference plane ${ }^{2,11,14,16)}$ and superior ${ }^{9)}$ and/or inferior endplate $e^{2,7,14)}$ were used as reference plane for sagittal angle of pedicle. Uncertainty and inaccuracy of measurement could be emerged because three dimensional position of vertebra could not be reflected using these reference plane. To consider the real position of each vertebra in each person and compare angular parameters of vertebra among person, constant part of vertebra with least variation should be used as the origin of reference and local coordinate. In our study, posterior wall of each vertebral body was used as the origin of local coordinate and reference plane. The transverse and sagittal angle of each vertebra in different position in each person was calculated firstly. These angles were transformed using the position of posterior wall of each vertebral body and could be compared among persons.

The absolute values of angle in our study were not same but the trend of changes was similar to previous studies ${ }^{12,15)}$. The sagittal angle could be easily monitored than transverse angle with intra-operative fluoroscopy, especially with oblique and lateral X-ray image. Cha et al. ${ }^{1)}$ reported that coaxial and

Table 1. The transverse angle of ideal trajectory of pedicle screw insertion from C3 to C6

\begin{tabular}{lccccccccc}
\hline Level & Rt $\left({ }^{\circ}\right)$ & Max & Min & Lt $\left({ }^{\circ}\right)$ & Max & Min & Total $\left({ }^{\circ}\right)$ & Max & Min \\
\hline C3 & $49.36 \pm 2.10$ & 61.46 & 16.17 & $51.84 \pm 05.69$ & 86.25 & 34.41 & $50.60 \pm 6.22$ & 86.25 & 16.17 \\
C4 & $51.04 \pm 3.01$ & 68.22 & 25.46 & $51.79 \pm 08.50$ & 74.23 & 41.00 & $51.42 \pm 7.44$ & 74.23 & 25.46 \\
C5 & $48.33 \pm 2.93$ & 76.55 & 32.47 & $47.26 \pm 14.91$ & 61.54 & 25.82 & $47.79 \pm 7.61$ & 76.55 & 25.82 \\
C6 & $41.76 \pm 7.83$ & 60.00 & 26.28 & $40.71 \pm 12.22$ & 61.71 & 21.70 & $41.24 \pm 7.76$ & 61.71 & 21.70 \\
\hline
\end{tabular}

Values are presented as mean \pm standard deviation or number. Rt : right, Lt : left, Max : maximum, Min : minimum

Table 2. The sagittal angle of ideal trajectory of pedicle screw placement from C3 to C6

\begin{tabular}{lccccccccc}
\hline Level & Rt $\left({ }^{\circ}\right)$ & Max & Min & Lt $\left({ }^{\circ}\right)$ & Max & Min & Total $\left({ }^{\circ}\right)$ & Max & Min \\
\hline C3 & $9.72 \pm 1.17$ & 18.67 & 1.92 & $9.71 \pm 05.05$ & 20.73 & -1.94 & $9.72 \pm 6.73$ & 20.73 & -1.94 \\
C4 & $4.36 \pm 2.71$ & 17.30 & -9.74 & $5.81 \pm 12.89$ & 18.10 & -7.02 & $5.09 \pm 6.39$ & 18.10 & -9.74 \\
C5 & $-0.44 \pm 1.46$ & 12.98 & -11.54 & $0.28 \pm 02.75$ & 15.15 & -11.19 & $0.08 \pm 6.06$ & 15.15 & -11.54 \\
C6 & $-3.60 \pm 4.08$ & 10.77 & -17.41 & $0.25 \pm 02.74$ & 15.49 & -10.77 & $-1.67 \pm 6.06$ & 15.49 & -17.41 \\
\hline
\end{tabular}

Values are presented as mean \pm standard deviation or number. Rt : right, Lt : left, Max : maximum, Min : minimum 
oblique fluoroscopy showed better sensitivity for superoinferior direction than mediolateral direction of cervical pedicle screws. The upper and lower margin of pedicle easily found in oblique views in practice. Three dimensional reconstruction of our model showed direction to superior corner of vertebral body is the best direction and it could be easily observed with lateral X-ray views but care should be paid not to penetrate superior endplate.

The various anatomical landmarks including parts of lateral mass $^{14)}$, superior and inferior articular process ${ }^{8,10)}$, lateral vertebral notch ${ }^{8,9)}$ used to describe optimal entry point of pedicle screw insertion. In our study, the entry point could be seen if central line of ideal trajectory fused with 3D cortical model image. They showed $2 \mathrm{~mm}$ directly medial to the lateral vertebral notch in a rough way like previous study ${ }^{9)}$. We are now trying to find anatomical landmark that have characteristics of easy to find, measure and describe in surgical field. Robotic pedicle screw insertion needs several essential considerations. First, Optimal analyzing methods for three dimensional morphology of pedicle is most important step and should be invented by medical doctors. In this study, we defined and measured ideal direction of cervical pedicle screw placement using in vivo 3D cortical shell model that contains individual anatomical characteristics. Because our results were measured using computing process for reconstructed 3D model, our method could be easily embedded in the computer program for robotic screw insertion. Second, Continuous positioning of spatial location of pedicle and navigation of screws based on morphology and changing location of pedicle also important and should be the roll of engineers. The limitation of our study is degeneration because reconstruction and analysis of aged bone easily induced committing errors.

\section{CONCLUSION}

We analyzed 3D direction of cervical pedicle screw placement using reconstructed cortical shell model from in vivo CT image data. Because we know 3D Cartesian coordinate of all points constituting cortical shell of cervical vertebrae. we can easily reconstruct and manage 3D model using computer program. Diverse morphological characteristics and individual anatomical variations could be easily analyzed similar to direct inspection of cadaveric specimen. Furthermore, our method could be easily used in the computing program for automated robotic screw insertion.

\section{CONFLICTS OF INTEREST}

No potential conflict of interest relevant to this article was reported.

\section{INFORMED CONSENT}

Informed consent was obtained from all individual participants included in this study.

\section{AUTHOR CONTRIBUTIONS}

\author{
Conceptualization : JSH, HYK \\ Data curation : JSH \\ Formal analysis : JHH, HGP \\ Funding acquisition : JSH \\ Methodology : JSH, HYK \\ Project administration : JSH, HYK \\ Visualization : HYK \\ Writing - original draft : JSH \\ Writing - review \& editing : JSH, HYK
}

\section{- Acknowledgements}

This work was supported by a research grant from Jeju National University Hospital.

\section{References}

1. Cha SH, Kim C, Choi BK, Kim HJ, Baek SY : C-arm assessment of cervical pedicle screw: screw coaxial fluoroscopy and oblique view. Spine (Phila Pa 1976) 32 : 1721-1727, 2007

2. Chanplakorn P, Kraiwattanapong C, Aroonjarattham K, Leelapattana P, Keorochana G, Jaovisidha $S$, et al. : Morphometric evaluation of subaxial cervical spine using multi-detector computerized tomography (MD-CT) scan: the consideration for cervical pedicle screws fixation. BMC Musculoskelet Disord 15 : 125, 2014

3. Hojo Y, Ito M, Suda K, Oda I, Yoshimoto H, Abumi K : A multicenter study on accuracy and complications of freehand placement of cervical 
pedicle screws under lateral fluoroscopy in different pathological conditions: CT-based evaluation of more than 1,000 screws. Eur Spine J 23 : 2166-2174, 2014

4. Ito Z, Higashino K, Kato S, Kim SS, Wong E, Yoshioka K, et al. : Pedicle screws can be 4 times stronger than lateral mass screws for insertion in the midcervical spine: a biomechanical study on strength of fixation. J Spinal Disord Tech 27 : 80-85, 2014

5. Jo DJ, Seo EM, Kim KT, Kim SM, Lee SH : Cervical pedicle screw insertion using the technique with direct exposure of the pedicle by laminoforaminotomy. J Korean Neurosurg Soc 52 : 459-465, 2012

6. Johnston $T L$, Karaikovic EE, Lautenschlager EP, Marcu D : Cervical pedicle screws vs. lateral mass screws: uniplanar fatigue analysis and residual pullout strengths. Spine J 6 : 667-672, 2006

7. Karaikovic EE, Daubs MD, Madsen RW, Gaines RW Jr : Morphologic characteristics of human cervical pedicles. Spine (Phila Pa 1976) 22 : 493-500, 1997

8. Karaikovic EE, Kunakornsawat S, Daubs MD, Madsen TW, Gaines RW Jr : Surgical anatomy of the cervical pedicles: landmarks for posterior cervical pedicle entrance localization. J Spinal Disord 13 : 63-72, 2000

9. Lee DH, Lee SW, Kang SJ, Hwang CJ, Kim NH, Bae JY, et al. : Optimal entry points and trajectories for cervical pedicle screw placement into subaxial cervical vertebrae. Eur Spine J 20 : 905-911, 2011
10. Liu J, Li Y, Wu Y, Zhu Q : A novel method of cervical pedicle screw placement from C3 to C5 and its clinical applications. Spine (Phila Pa 1976) 38 : E504-E512, 2013

11. Ludwig SC, Kramer DL, Balderston RA, Vaccaro AR, Foley KF, Albert TJ : Placement of pedicle screws in the human cadaveric cervical spine: comparative accuracy of three techniques. Spine (Phila Pa 1976) 25 : 1655-1667, 2000

12. Panjabi MM, Duranceau J, Goel V, Oxland T, Takata K : Cervical human vertebrae. Quantitative three-dimensional anatomy of the middle and lower regions. Spine (Phila Pa 1976) 16 : 861-869, 1991

13. Panjabi MM, Shin EK, Chen NC, Wang JL : Internal morphology of human cervical pedicles. Spine (Phila Pa 1976) 25 : 1197-1205, 2000

14. Ruofu Z, Huilin Y, Xiaoyun H, Xishun H, Tiansi T, Liang C, et al. : CT evaluation of cervical pedicle in a Chinese population for surgical application of transpedicular screw placement. Surg Radiol Anat 30 : 389-396, 2008

15. Tan SH, Teo EC, Chua HC : Quantitative three-dimensional anatomy of cervical, thoracic and lumbar vertebrae of Chinese Singaporeans. Eur Spine J 13 : 137-146, 2004

16. Uğur HC, Attar A, Uz A, Tekdemir I, Egemen N, Cağlar S, et al. : Surgical anatomic evaluation of the cervical pedicle and adjacent neural structures. Neurosurgery 47 : 1162-1168; discussion 1168-1169, 2000 